Journal of Engineering and Applied Sciences 15 (1): 192-197, 2020

ISSN: 1816-949X

(C) Medwell Journals, 2020

\title{
Development of Miniature Base Transceiver Station using Radio Frequency Energy Harvesting
}

\author{
${ }^{1}$ Win Adiyansyah Indra, ${ }^{1}$ Z. Manap, ${ }^{2}$ Adi Saptari and ${ }^{1}$ Nurin Alya Binti Ismail \\ ${ }^{1}$ Centre for Telecommunication Research and Innovation, \\ Fakulti Teknologi Kejuruteraan Elektrik and Elektronik, \\ Universiti Teknikal Malaysia Melaka, Hang Tuah Jaya, \\ 76100 Durian Tunggal, Melaka, Malaysia \\ ${ }^{2}$ Department of Industrial Engineering, President University, Bekasi, Indonesia
}

\begin{abstract}
This study developed miniature of base transceiver station powered up by radio frequency energy harvesting. Base transceiver station is one of the major equipment in telecommunication system that able to transmit the signal to the receiver such as mobile phones which let it to complete the communication process. However, the base station need to have power supply to powered up the equipment. Previously studied, the base station's power supply was from nature sources such as solar energy or wind energy. Due to the unstable condition of the weather, the power radiated might not be able to power up the base station hence, RF energy harvested was introduced. There are 3 main objectives of the project, firstly is to develop the basic concept of base transceiver station with radio frequency energy harvesting. Second, to implement the hall effect sensor into the concept of the base transceiver station. Third, to analyze the idea of the miniature of base transceiver station by look through the output and radio frequency energy harvesting. The RF energy harvested shows one of the methods to overcome this problem. RF energy harvested are one of the tools that need low frequency signal for it to transmit the power to the base transceiver station. The LEDs as the output which indicated as the antenna on the base transceiver station lights up as the hardware connection being made. Hence, the circuit for the RF energy harvesting and the hall sensor effect that will light up the LEDs shown. This study will show the implementation of the RF energy harvesting to the equipment.
\end{abstract}

Key words: Base transceiver station, radio frequency, energy harvesting, telecommunication, LEDs

\section{INTRODUCTION}

Base transceiver station or also known as the base station which is a tower functions as a mobile base station that transmits or receives the signal and emerging in cell area. The base transceiver station able to handle certain calls at a moment. A base station consists of 168 voice channels available and when the user is entering to another certain area, the base station will hand off to the other base station. However, the base station has its boundary to the amount of bandwidth channel that accessible for internet and data of a mobile user. The carrier needs to increase the channel bandwidth and the base stations to make sure that internet will not slow at peak hours (Raisa et al., 2016). Typical cell tower made up of a few parts such as antenna, supporting structure, some hardware and a link. These parts play a major role to build up the base transceiver station or the base station as we can see along the roads or highways. The function of the antenna is to transmit and receives the signal in the cell while the link is usually the wireless connections. The supporting structure is function where the antennas are attached while the hardware is to support the tower of the base stations which usually kept in a cabinet.

The output power with the environments such as trees, hills and building of the base station also affect the cell coverage area. For city areas, more base stations will be seen with the number of cell sizes is $2-5 \mathrm{~km}$ as there are large number of users and barriers. However, the cell radius will be positioned 10-32 km which quite longer distance if it is in large open spaces. The purpose of the antenna to be built up at a tower is because of it makes it easier to stay in communication with the users such as the cell phone users and also to overcome any blockades for instance like buildings or trees that were situated in between those base stations. In Malaysia, Telekom Malaysia Berhad (TM) is involved for the formation, maintenance and provide telecommunications services (Al-Falahy and Alani, 2017). On the other hand, Energy Harvesting (EH) is functioned as it derives energy of outside sources such as the solar power, thermal energy, kinetic energy and many more and then stores it for length

Corresponding Author: Win Adiyansyah Indra, Centre for Telecommunication Research and Innovation,

Fakulti Teknologi Kejuruteraan Elektrik and Elektronik, Universiti Teknikal Malaysia Melaka,

Hang Tuah Jaya, 76100 Durian Tunggal, Melaka, Malaysia 
of time and finally transmit the power or give electricity to devices wirelessly. Specifically, for Radio Frequency (RF) energy harvesting, it provides low-voltage and low power to its application in wide range of mobile market such as medical equipment or transportation. This type of radio frequency of energy harvesting also beneficial for applications that entail back-up battery specifically in area that is hard to reach (Sapakal et al., 2013). This energy can be obtained from different wasted sources which also known as energy scavenging that take the residual energy which release to the surrounding becoming waste, turned as a byproduct of nature environmental phenomenon. In addition, the energy used improve the efficiency for example, by cut the cost if the energy harvested use to help to power up the devices. Other than that, it allows latest technology for instance wireless sensor networks.

There are some advantages of using RF-EH such as it is maintenance free which it does not need to replace batteries after the voltage of the batteries run out. Secondly, it is environment friendly this is because the usage of batteries reduces as it contains chemical stuff which are harmful and unsafe to surrounding. Lastly, it gives latest technology to the telecommunication industry such as sensors to show or detect isolated areas (Choi et al., 2016).

Base Transceiver Station (BTS): Base Transceiver Station (BTS) can also be known as radio base station is equipment that enables the user on the device and operator of a network in order to be able to wirelessly communicate to one another. The device of the user can be mobile phones, laptops which can connect to a wireless internet. Meanwhile the examples for the operator network are the Global System of Mobile Communication (GSM), Wi-Fi along with others. In Fig. 1, shows the equipment of base transceiver station which entails Direct Current (DC) power supply input (Agrawal et al., 2016).

The Base Transceiver Station (BTS) contain a sole rack or cabinet which locate the importance aspects of a point to multi-point RF communication network. It's explain that the telecommunication sector that take part in a major part of developing the economy through globally and for the information or knowledge to spread (Tinkhede and Ingole, 2014).

It explains that in future, the mobile cellular network field is accountable to the consumption of the energy along with the number of radiations it releases to surrounding. In conjunction of the application that is not depending due to the electric supply, the off-grid Base Stations (BSs) normally would be use because of its capability to deliver radio coverage through varied topographical part (Shurman et al., 2012).

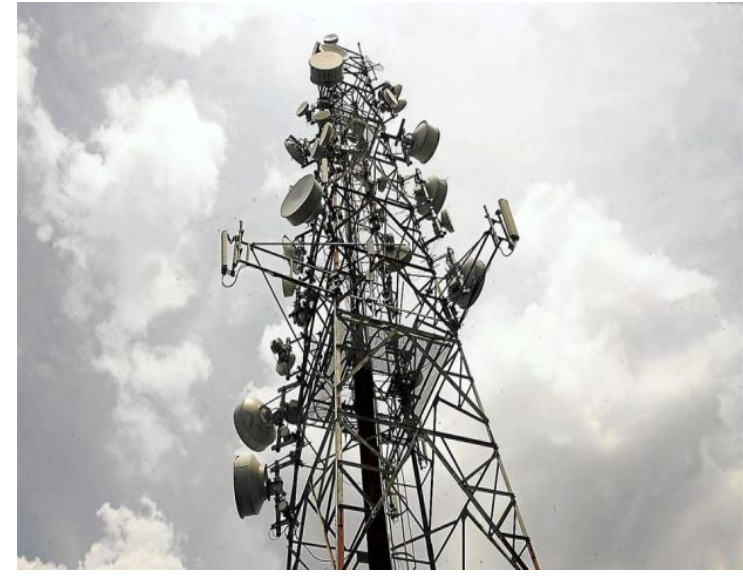

Fig. 1: Example of base transceiver station tower

Base transceiver station represent one of the telecommunication systems which transmits signals for a certain distance. In telecommunication, it involved in operations, maintenance and provides access for the data, voices, text and sound. The ICT industry, especially, showed the most rapidly growing sectors in mobile telecommunications industry. It explains that the first mobile phones were large and bulky. However, as the time goes by $1 G$ cellular operator from Celcom was introduced as it starts year of 1989 through the primary of automatic radio telecommunication 900 (ART 900) regarding to the British extended total access communications system technology. In this system, measurement of the user's telephone converted significantly lesser in size hence make it look more suitable towards the years.

In the study of Yifei and Longming (2014), they proposed that network become whichever wireless technology for example the Global System for Mobile communication (GSM) or even Wi-Fi. Nevertheless, since, BTS is related to the telecommunication, it denotes to appliances that produces "cell” in a cellular network. However, the whole base station is unsuitably mentioned as BTS or the mobile phone pillar. The BTS consist equipment to encryption along with decryption of the communication or infrastructures, range clarifying equipment, the antennas with the transceivers. The base transceiver station normally takes many transceivers which let it to assist other cell's different frequencies and sectors.

In this era, software technology and programming languages are continuously emerging, hence, inventive methods to software design along with the growth of Information Technology (IT) industry are continuously explored. Kumar et al. (2015) explained that the important responsibility for BTS platform Software (SW) is to hide the hardware environment for the software 


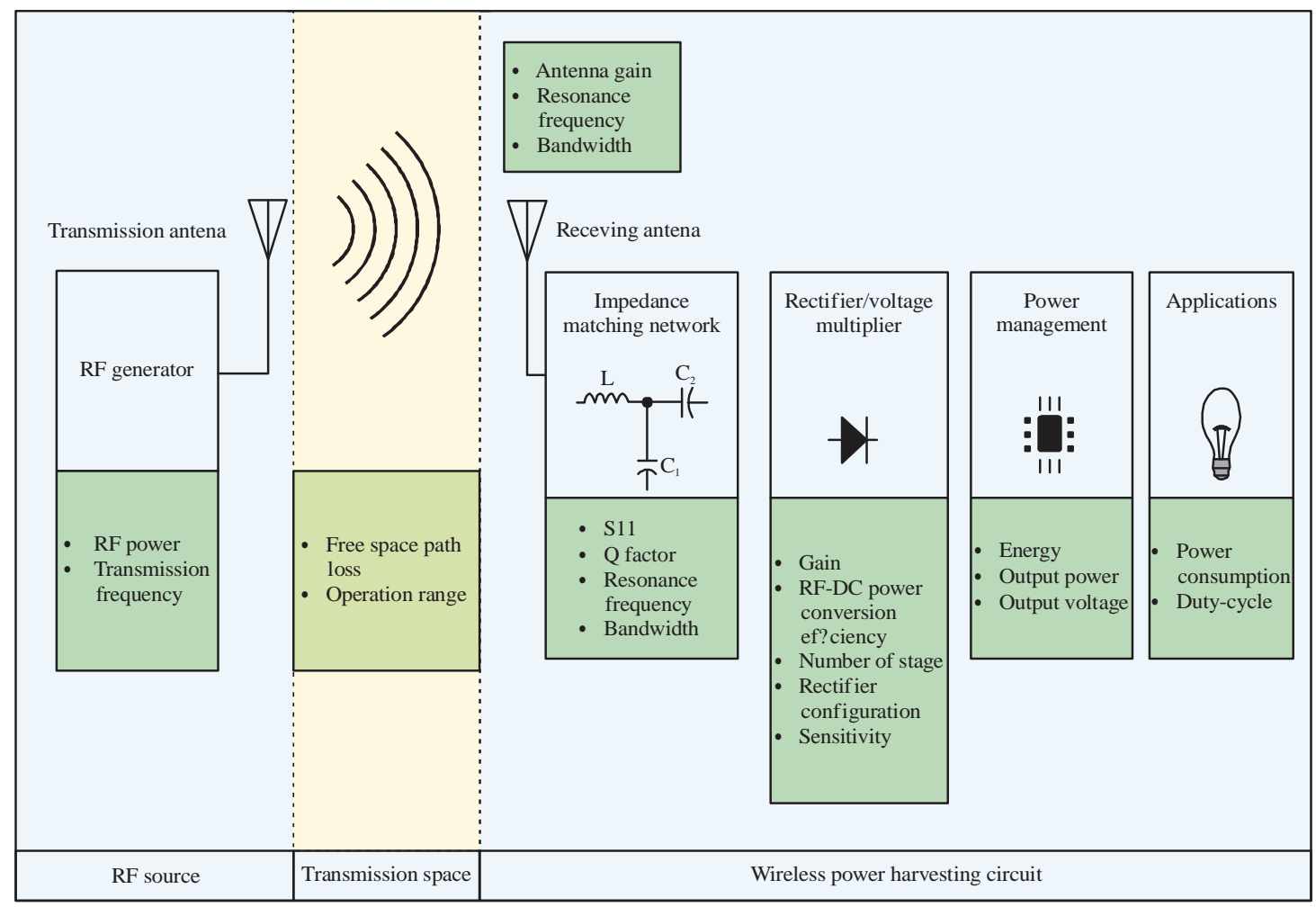

Fig. 2: Block diagram of RF energy harvesting system

applications. Figure 2 presents the SW modularization concept. Network Element (NE), for example the LTE BTS is a mixture of software and hardware and it involves of diverse System Components (SC) and System Elements (SE).

RF energy harvesting: For the energy harvesting (Miyim et al., 2014) proposed that the method possibilities higher opportunity to the replacement for minor battery in low power electrical devices along with the systems. RF Wireless Power Harvesting (WPH) holds huge possible to replace battery or to higher up the lifespans. Recently, batteries power a majority of low-power remote sensor devices and embedded equipment. Jain et al. (2018) proposed that energy harvesting can convert dissipated energy turned to electricity which able to be a battery recharger for environment sources or the autonomous power sources. In addition, Gong et al. (2018) state, the energy harvesting as from renewable energy sources such as solar or wind energy that produce electricity for power up the off-grid BSs shows the robust selections in order for it to power up the BSs regarding on their plentiful accessibility for higher spectrum due to the geographic area globally. But because of the random and irregular nature such as wind and solar, the operation running for the sources basically has to be integrated to any means of renewable or non-renewable power supply as well as for the energy storage solutions as it to let the power supply in a BS continue to power up.

In addition, Ahmad et al. (2017) state, the energy harvesting as from renewable energy sources such as solar or wind energy that produce electricity for power up the off-grid BSs shows the robust selections in order for it to power up the BSs regarding on their plentiful accessibility for higher spectrum due to the geographic area globally. But because of the random and irregular nature such as wind and solar, the operation running for the sources basically has to be integrated to any means of renewable or non-renewable power supply as well as for the energy storage solutions as it to let the power supply in a BS continue to power up.

The harvesting energy is an unlimited operating lifespan which is good for maintaining the operation. As Indra et al. (2019a, b) proposed that, for battery it has smaller lifespans as well as it needs continuous replacement. Hence, by applying power harvesting technologies, devices and equipment, this would convert self-sustaining for energy needed to the system, hence receiving an unlimited operating lifespan. By this, request for power conservation would be insignificant. Mandour et al. (2019) also proposed that the wireless power transmission researched for a lot of years as a means to replace or complement battery, hence, identified the energy independent devices through other applications. 
After that Gong et al. (2018) explain that, for it to assess the viability of deploying ambient RF energy harvester, accessible RF power requires to measure for different areas. The measurement along with data on harvester performance, hence, this be able to identify the locations due to where RF harvester powered successfully deployed.

\section{MATERIALS AND METHODS}

Base transceiver station is the main component in completing this project. The base station is functioned as the devices that receives and transmit the signal to the device related such as mobile phone. For this project, the miniature base station receives its input from the RF energy harvesting and transmit it to the output which is the LED. The miniature of the prototype of the wireless base station was set up such that it consists of a tower that almost signify like the actual base transceiver station and the LEDs as the antenna which it signifies the output of the project. The tower of the base transceiver station that use is a miniature as the example tower that includes the LEDs which represent as the antenna connected or attached to it.

In this project, the Arduino Uno R3 plays an important role for the system to work. The Arduino Uno operated as it is the device that allows the presence of the LEDs as the output by code the program. In this system, the Arduino will be connected to the hall effect sensor circuit. The coding is written and also it generated in the IDE software which is specifically for the Arduino Software. The type of language used are $C$ language.

For RF power harvester is based on the P2110 RF harvester receiver ICs where it converts $915 \mathrm{MHz}$ RF received using a patch antenna which gain $=6 \mathrm{dBi}$ towards DC, hence, it stores it in a super capacitor of $33 \mathrm{mF}$. When a charge threshold $(1.25 \mathrm{~V})$ on the capacitor is achieved, the P2110 boosts the voltage to the set output voltage level (3.3 or $4.2 \mathrm{~V}$ ) and enables the voltage output. When the capacitor voltage goes below $1.02 \mathrm{~V}$, P2110 shut off its output. Capacitor will recharge again, and the cycle will repeat. There are some components involve such as the transmitter (TX9150), a wireless sensor network harvester and radio along with network monitor that connected to the PC. The are some advantages such that it is high conversion efficiency and also converts low level RF signals. The RF energy circuit was constructed as shown in the stripboard in Fig. 3 according to the uses of capacitors and diodes. After that, the lithium 3.7 rechargeable battery are connected after the charging board as to store the energy before transmitting to the circuit of the BTS.

Hall effect sensor of 44E 402 consists of 3 legs which represents the VCC $(5+)$, ground in the middle and the digital output. The electromagnetic based on the principle of when current pass through hence magnetic field will

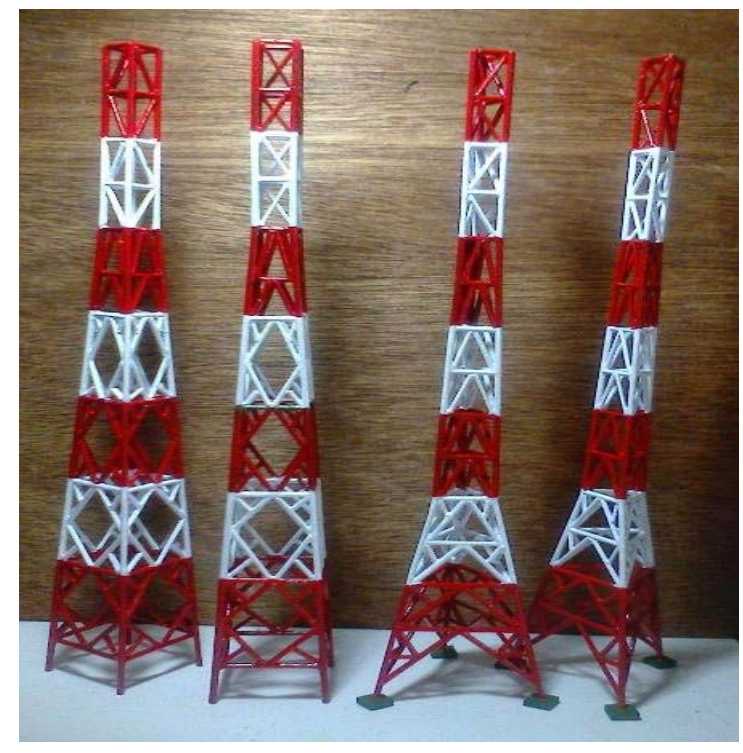

Fig. 3: Miniature of BTS tower

occurred surround it. As the current supplied, the presence of a magnet in front of it will indicate as ON however, if the magnet is away it will indicate as OFF.

For the digital output, it can be divided into different types of power supply such as the regulated and unregulated. For the regulated, the ranges of the power supplied can be from 3.8-24 V. However, the unregulated can be used for special application which needs DC supply between $4.5-5.5 \mathrm{~V}$. These must be conjunction to the logic circuits in need of $5 \mathrm{~V}$ supplied power to it.

The signal send from the radio frequency were observed and analyses. The frequency transmitted is able for the base transceiver station to power up hence giving the output LED to light up. Besides, the voltages and the distance of the magnet to the hall effect sensor to were also taken. The preliminary result of the project can be estimated as the RF energy harvesting was transmitting energy for the Arduino Uno as its operating voltage is $5 \mathrm{~V}$ and hence to output which are the LEDs.

\section{RESULTS AND DISCUSSION}

The frequency from a transmitter were supplied to the radio frequency energy harvesting circuit which consists of capacitors, resistors and diodes. From the circuit, it is then connected to the charging board then stored in the lithium rechargeable battery of $3.7 \mathrm{~V}$ of $600 \mathrm{mAh}$. After that, the lithium rechargeable battery was connected to the Arduino Uno circuit which consists of the hall effect sensor. The LEDs that act as the output light up as soon as the magnet placed in front of the hall effect sensor (Fig. 4).

The results of the project can be seen as firstly, the RF energy harvesting can operate in the circuit where it can later store the energy in the rechargeable battery. The 


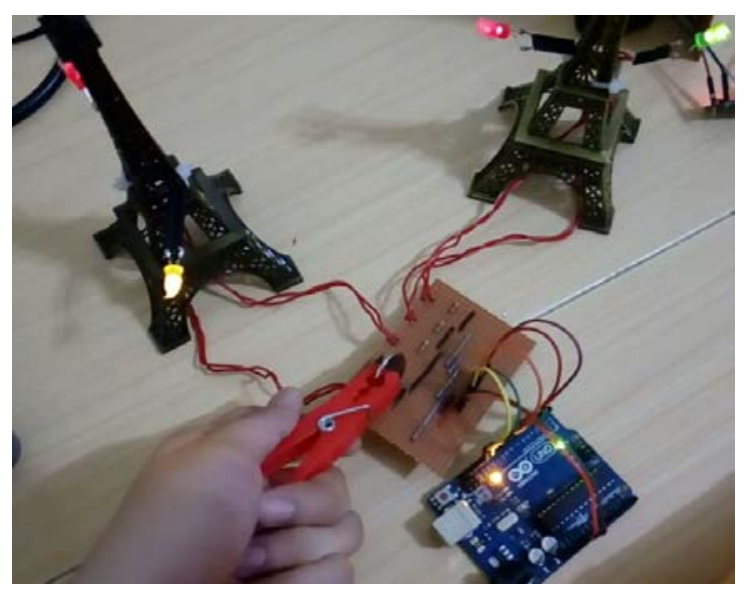

Fig. 4: Connection of hall effect sensor with presence of magnet

second result also can be identified when the LEDs light up as soon as the magnet is near to the hall effect sensor. The magnet represented as the users on the road and the LEDs represent the antennas from the base transceiver station. The data analysis based from the project conducted, shows that, the voltage from the RF energy harvesting circuit before during and after the frequency were transmitted. The voltage and current obtained for RF circuit with load were $0.697 \mathrm{~V}$ and $0.695 \mathrm{~mA}$, respectively. The reading was taken at different places for several times and the average reading were also calculated accordingly. The further the magnet to the sensor the higher chances of loss connection for the LED to light up. Same goes as it apply to the user, the further to the BTS the lesser connection or no connection to the mobile phone.

The results of the project conducted regarding to the development of miniature of base transceiver station using radio frequency energy harvesting shows that the LEDs are able to light up. The LEDs indicating the output of the base transceiver station lights up as the power was supplied based from the RF energy harvesting circuit. The purposes of LEDs in this system are functioned as the antenna to the base transceiver station. The antennas used are the Omni antenna which the Red LEDs at the circuit represented. Yellow LED represent the directional antenna while green LED represent the Helical antenna. For the Omni antenna, it is can also known as the round and circle type of surfaces in real life. Omni antenna is an antenna that can transmits or receives the signal with $360^{\circ}$ of direction, in other word, available for any direction within the horizontal plane. Sector Antenna or the directional microwave antenna is an antenna that can only transmits or receive in one specific direction such as for 60, 90 and $120^{\circ}$. Meanwhile for Helical antenna, it is an antenna that has one or more conducting wire that is wound helical shape which can distributes for multi-dimensional signal.
The main component used in this project are the RF energy harvesting circuit which consists main component such as capacitors and diodes. From the circuit it stores the energy inside the lithium rechargeable battery of 3.7 $\mathrm{V}$ and transmits to the hall effect sensor circuit. The Hall Effect Sensor circuit operates with Arduino Uno R3 to let the LEDs to light up. The main function of the Arduino Uno used in this project is to code the operation for the hall effect sensor.

Hall effect sensor is a magnetic field sensor that operates when there is presence of a magnet. This sensor includes means of biasing, offset reduction and temperature compensation. They are two types of operation of the hall effect sensor such that, one is for analog and another one is digital. These types are to determine the output by different coding as in this project uses digital hall effect sensor which the output only stated as 0 or 1 . Furthermore, there are also various hall effect sensor that can be found with different specifications. In this project, as the magnet approach nearer to the sensor, the LEDs light up by means that as the user getting nearer to the BTS, more signal they will receive. From the data received in Table 4.4 shows that the distance is proportional to the detection of the signal. At $0 \mathrm{~cm}$ until $2 \mathrm{~cm}$ the users can still receive the signal meanwhile as entering $2.5 \mathrm{~cm}$ the signal cannot be detected.

As the conclusion, to make system in this project works, the RF circuit and sensor both need to be operate. From the coding that had been done in Arduino IDE, the circuit can be operated. Hence, the results in this project can be seen at the LEDs as it can be light up. There are a few limitations for this project to work during handling this project such as to identify the voltages of the RF energy harvesting circuit can supply for the limited appliances, with the shorter range of distance. This is because the transmission received are not strong. Other than that, by using RF energy harvesting circuit, it must use the antenna especially direct antenna to receive the optimum signal to the circuit. Lastly, the actual magnitude regarding to the real time BTS are not able to determine by this system. Signals at the LEDs can only be detected by the motion of the magnet, however, it cannot be measure by the actual magnitude. Hence, this needs to be improved as for the system can be used for user in the future.

\section{CONCLUSION}

The whole concept of the miniature of base transceiver station which were the mini tower along with the LEDs can be light up. These LEDs were representing as the antenna that it received the power supply from the RF energy circuit hence light up indicating that there is a connection. Other than that, the main purpose for this project is to look at how much energy from the RF energy harvesting circuit can generate for it to supply to the 
Arduino Uno and hall effect sensor. The RF energy harvesting consists of advantages such as it can be power up anytime and anywhere. In our unstable climate and weather, RF energy harvesting also can be applied in indoors compared to the solar energy or wind energy. By this, users can receive the networks by using any kind or technology without any problems.

\section{ACKNOWLEDGEMENT}

The researchers would like to thank Universiti Teknikal Malaysia Malacca (UTeM) for sponsoring this work under the Grant PJP/2019/FTKEE (4B)/S01656.

\section{REFERENCES}

Agrawal, A., A. Jeyakumar and N. Pareek, 2016. Comparison between vertical handoff algorithms for heterogeneous wireless networks. Proceedings of the 2016 International Conference on Communication and Signal Processing (ICCSP), April 6-8, 2016, IEEE, Melmaruvathur, India, ISBN:978-1-4673-8549-7, pp: 1375-1373.

Ahmad, R., E.A. Sundararajan, N.E. Othman and M. Ismail, 2017. Handover in LTE-advanced wireless networks: State of art and survey of decision algorithm. Telecommun. Syst., 66: 533-558.

Al-Falahy, N. and O.Y. Alani, 2017. Technologies for 5G networks: Challenges and opportunities. IT Prof., 19: 12-20.

Choi, Y.I., J.H. Kim and N.I. Park, 2016. Revolutionary direction for 5G mobile core network architecture. Proceedings of the 2016 International Conference on Information and Communication Technology Convergence (ICTC), October 19-21, 2016, IEEE, Jeju, South Korea, ISBN:978-1-5090-1326-5, pp: 992-996.

Gong, F., Z. Sun, X. Xu, Z. Sun and X. Tang, 2018. Cross-tier handover decision optimization with stochastic based analytical results for 5G heterogeneous ultra-dense networks. Proceedings of the 2018 IEEE International Conference on Communications Workshops (ICC Workshops), May 20-24, 2018, IEEE, Kansas City, Missouri, USA., ISBN:978-1-5386-4329-7, pp: 1-6.

Indra, W.A., M.S. Hamid, N.B.M. Yusof, N. A/P Jayraman and H. Rusnandi, 2019b. Frequency reuse optimization for OFDMA network. J. Eng. Appl. Sci., 14: 1219-1225.

Indra, W.A., S. A/L Sukumaran, K.A.M. Annuar and Irianto, 2019a. Analysis of handover planning in Wideband Code Division Multiple Access (W-CDMA). J. Eng. Appl. Sci., 14: 823-830.
Jain, A., E. Lopez-Aguilera and I. Demirkol, 2018. Improved handover signaling for $5 \mathrm{G}$ networks. Proceedings of the 2018 IEEE 29th Annual International Symposium on Personal, Indoor and Mobile Radio Communications (PIMRC), September 9-12, 2018, IEEE, Bologna, Italy, ISBN:978-1-5386-6010-2, pp: 164-170.

Kumar, S., G. Gupta and K.R. Singh, 2015. 5G: Revolution of future communication technology. Proceedings of the 2015 International Conference on Green Computing and Internet of Things (ICGCIoT), October 8-10, 2015, IEEE, Noida, India, pp: 143-147.

Mandour, M., F. Gebali, A.D. Elbayoumy, G.M.A. Hamid and A. Abdelaziz, 2019. Handover optimization and user mobility prediction in LTE femtocells network. Proceedings of the 2019 IEEE International Conference on Consumer Electronics (ICCE), January 11-13, 2019, IEEE, Las Vegas, Nevada, USA., ISBN: 978-1-5386-7911-1, pp: 1-6.

Miyim, A.M., M. Ismail and R. Nordin, 2014. Vertical handover solutions over LTE-advanced wireless networks: An overview. Wireless Personal Commun., 77: 3051-3079.

Raisa, F., A. Reza and K. Abdullah, 2016. Advanced inter-cell interference management technologies in 5G wireless Heterogeneous Networks (HetNets). Proceedings of the 2016 IEEE Student Conference on Research and Development (SCOReD), December 13-14, 2016, IEEE, Kuala Lumpur, Malaysia, ISBN: 978-1-5090-2949-5, pp: 1-4.

Sapakal, M.R.S. and M.S.S. Kadam, 2013. 5G mobile technology. Intl. J. Adv. Res. Comput. Eng. Technol., 2: 568-571.

Shurman, M.M., M.F. Al-Mistarihi and S.A. Nasser, 2012. Hard handover optimization in mobile WiMAX networks. Proceedings of the 5th International Conference on Communications, Computers and Applications (MIC-CCA2012), October 12-14, 2012, IEEE, Istanbul, Turkey, ISBN:978-1-4673-5230-7, pp: 148-153.

Tinkhede, P. and P. Ingole, 2014. Survey of handover decision for next generation. Proceedings of the International Conference on Information Communication and Embedded Systems (ICICES2014), February 27-28, 2014, IEEE, Chennai, India, pp: 1-5.

Yifei, Y. and Z. Longming, 2014. Application scenarios and enabling technologies of 5G. China Commun., 11: 69-79. 